for any deep sedation, however many hospital paediatricians have the skills required to administer such a drug ${ }^{2}$ providing they have experience with the drug and are familiar with its complications and contraindications.

1 Cote CJ. Sedation for the pediatric patient - a review. Pediatr Clin N Am 1994; 41: 31-58.

2 American Academy of Pediatrics. Committee on drugs. Guidelines for monitoring and management of pediatric patients during and afte sedation for diagnostic and therap

\section{Carbon monoxide poisoning in two children riding in the back of a van}

EDITOR,-A brother and sister aged 9 and 10 years who were both previously fit and well were noticed to be abnormally drowsy after a 40 minute journey in the back of a Transit type van. On arrival at the accident and emergency department, both children were drowsy but orientated in time and space. Both complained of headache and both had vomited. Neither had abnormal neurological signs on examination.

Arterial blood gases revealed carboxyhaemoglobin ( $\mathrm{COHb}$ ) concentrations of $24.5 \%$ and $19 \cdot 7 \%$ respectively by absorbtion photometry (International Laboratories 482 Co Oximeter). Both children were treated with $100 \%$ inspired oxygen at 6 litres/minute via a well fitting face mask. Symptomatic improvement was apparent at one hour and both were fully alert with normal intellectual function at two hours. Repeat blood gases six hours after admission showed $\mathrm{COHb}$ concentrations of less than $1 \%$ (normal range in urban non-smokers $<2 \%$ ). Three months after the poisoning, both children were reassessed. At this stage, there were no detectable intellectual or behavioural abnormalities noted by either parents or teachers. No neurological deficits were present.

Admission levels of $\mathrm{COHb}$ correlate poorly with short or long term sequelae. ${ }^{1}$ However, the levels measured in these children have been associated with long term neuropsychiatric morbidity in some patients. Treatment with hyperbaric oxygen is recommended if $\mathrm{COHb}$ concentrations exceed $40 \%$ or if there is a history of loss of consciousness, persisting neurological or inte lectual deficits, or cardiovascularabnormalities. ${ }^{2}$ None of these features was present in these two cases and both made a complete recovery. However, had the journey been of longer duration or the symptoms not recognised, a serious or fatal poisoning may have occurred.

The dangers of riding in the back of 'pick-up' trucks has recently been highlighted. ${ }^{3}$ In a series of 68 consecutive carbon monoxide poisonings, 20 occurred in children travelling in the back of pick-up type trucks, beneath canopies or soft covers, with defective or modified exhaust systems. In this case, the children were travelling in the back of a fully enclosed van. Subsequent inspection by the owner revealed a small crack in the exhaust system.

Carbon monoxide poisoning is a common cause of fatal poisoning. Clinicians must maintain a high degree of suspicion to recognise cases who frequently present with non-specific signs. Travelling in a vehicle with a damaged or non-standard exhaust system is a significant risk factor and travelling in the back of vans may be an additional risk for carbon monoxide poisoning.

$$
\begin{array}{r}
\text { R A SMITH } \\
\text { R J BALL } \\
\text { York District Hospital, } \\
\text { Wigginton Road, } \\
\text { York YO3 } 7 H E
\end{array}
$$

1 Meredith T, Vale JA. Carbon monoxide poisoning. $B M \mathcal{F}$ 1988; 296: 77-8.

2 Hampson NB, Norkool DM. Carbon monoxide poisoning in children riding in the back of pickup

3 Go Gorman DF, Clayton D, Gilligan JE, Webb RK. A longitudinal study of 100 consecutive admissions for carbon monoxide poisoning to the Royal Adelaide Hospital. Anaesth Inten Care 1992; 20: 311-6.

\section{Quality of life in surgically palliated complex congenital heart disease}

EDITOR,-Casey et al's review of patient after surgical palliation of complex congenital heart disease identifies the need to assess health status in children with chronic ill health. ${ }^{1}$ The ability to measure 'health related quality of life' (HRQL) in those with morbidity secondary to their disease, and any interventions performed by their clinicians, is increasingly being recognised as an essential facet of total patient care.

A comprehensive identification of the core set of attributes which combine to provide an index of HRQL revealed the following key set of six: sensory and communication ability, happiness, self care, pain or discomfort, learning and school ability, and physical activity. ${ }^{2}$ The Feeny-Barr multiattribute health assessment ${ }^{34}$ uses these domains to provide a utility score of HRQL and is a simple, concise instrument which may well be an appropriate tool to measure the overall morbidity burden in patients with chronic conditions such as cardiac patients. This instrument's practicability and validity is currently being evaluated in Nottingham and Canada.
ADAM GLASER DAVID WAI KER Department of Child Health University of Nottingham, Queen's Medical Centre, Nottingham NG7 $2 U H$

1 Casey FA, Craig BG, Mulholand HC. Quality of ife in surgically palliated complex congenital heart disease. Arch Dis Child 1994; 70: 382-6. 2 Cadman D, Goldsmith C, Bashim PP. Values, preferences and decisions in the care of children with developmental disabilities. $f$ Dev Behav Pediatr 1984; 5: 60-4.

3 Barr RD, Mohan KRP, Weitzmann S, et al. A multi-atrribute approach to health status multi-attribute approach to health status measurement and clinical management-illustrated by an application to brain tumours in 1994; 4: 639-48.

4 Billson AL, Walker DA. Assessment of health status in survivors of cancer. Arch Dis Child 1994; 70: $200-4$.

Central nervous system tumours lack national studies

EDITOR,-Thorne and Foreman raise a very important point when they highlight low entry of children with central nervous system $N$ tumours to national or international studies 음 which for other diseases have clearly been demonstrated to confer considerable benefit for the sufferers. ${ }^{1}$ The Medical Research Council does not organise solid tumour protocols for children apart from the joint project on bone tumours. The United Kingdom Children's Cancer Study Group (UKCCSG), however, has a range of protocols already in operation or in the planning stage for a variety of different central nervous system tumours including primitive neurectodermal tumour (medulloblastomas), brain stem gliomas, infants under 3 years with brain tumours, and, jointly with the International Society of Paediatric Oncology, a low grade glioma protocol. The problem is not the availability of protocols. Why paediatric oncologists, radiotherapists, and overwhelmingly neurosurgeons fail to participate in these studies is difficult to understand. The UKCCSG alone has run or actively participated in seven brain tumour studies since 1978 , as many as for any tumour group. What the authors need to do along with the rest of us is to try to ascertain why we cannot persuade specialists in other disciplines to actively participate in the available studies.

O B EDEN $D$ Academic Department of Paediatric Oncology, Fourth Floor, 38 Little Britain, St Bartholomew's Hospital, N London EC1A $7 B E$

1 Thorne R, Foreman NK. Central nervous system $N$ tumours lack national studies [letter]. Arch Dis $O$ Child 1994; 71: 95-6.



